\title{
The colored Jones polynomials of the figure-eight knot and the volumes of three-manifolds obtained by Dehn surgeries
}

\author{
by \\ Hitoshi Murakami (Tokyo)
}

\begin{abstract}
I describe how the colored Jones polynomials of the figure-eight knot determine the volumes of the three-manifolds obtained by Dehn surgeries along it, according to my joint work with Y. Yokota.
\end{abstract}

1. Introduction. In $1995 \mathrm{R}$. Kashaev defined a series of complex-valued link invariants parameterized by a natural number $N$ by using the quantum dilogarithm [7]; in 1997 he also proposed a conjecture that for any knot his invariants would give the hyperbolic volume of its complement if it possesses a complete hyperbolic structure [8]. J. Murakami and I proved that Kashaev's invariant parameterized by $N$ is the absolute value of the colored Jones polynomial associated with the $N$-dimensional irreducible representation of $s l_{2}(\mathbb{C})$ evaluated at an $N$ th root of unity [16]. We also generalized Kashaev's conjecture to the following Volume Conjecture.

ConjeCture 1.1 (Volume Conjecture [16]). Let $K$ be a knot in $S^{3}$ and $J_{N}(K ; t)$ its colored Jones polynomial associated with the $N$-dimensional irreducible representation of $s_{2}(\mathbb{C})$. Then

$$
2 \pi \lim _{N \rightarrow \infty} \frac{\log \left|J_{N}(K ; \exp 2 \pi \sqrt{-1} / N)\right|}{N}=v_{3}\left\|S^{3} \backslash K\right\| .
$$

Here $\left\|S^{3} \backslash K\right\|$ is the Gromov norm (or simplicial volume) [2]. It is the sum of the hyperbolic volumes of the hyperbolic pieces of $S^{3} \backslash K$ divided by $v_{3}$, where $v_{3}$ is the volume of the ideal regular ideal tetrahedron. So if $S^{3} \backslash K$ itself is hyperbolic then $v_{3}\left\|S^{3} \backslash K\right\|$ is equal to its hyperbolic volume.

2000 Mathematics Subject Classification: Primary 57M27, 57M50.

Key words and phrases: knot, figure-eight knot, colored Jones polynomial, volume, Dehn surgery, volume conjecture.

This research is partially supported by Grant-in-Aid for Scientific Research (B) (15340019). 
For more information about the volume conjecture and its further generalizations, see $[3,5,6,9,12,14,17,21,24-27]$.

Given a knot and a rational number one can define a closed threemanifold by Dehn surgery. For an integral Dehn surgery, one can calculate the Witten-Reshetikhin-Turaev (WRT) invariant as a linear combination of the colored Jones polynomials of the knot [20, 10]. In [13], I calculated fake limits (optimistic limits) of the WRT invariants of three-manifolds obtained by integral Dehn surgeries along the figure-eight knot and gave numerical data giving the volumes and the Chern-Simons invariants of the three-manifolds. This suggests that the colored Jones polynomials of a knot could give the volumes and the Chern-Simons invariants of three-manifolds obtained by Dehn surgeries along the knot.

In [15], I calculated the limit of the colored Jones polynomial of the figure-eight knot evaluated at $\exp (2 \pi r \sqrt{-1} / N)$ with $5 / 6<r<7 / 6$. It turned out that it gives the volume of a cone manifold with singularity along the figure-eight knot. Moreover in [18], Y. Yokota and I proved that this evaluation gives the volume and the Chern-Simons invariant even when $r$ is a complex number.

The purpose of this article is to describe the results in [18] with rough proofs and a short introduction to hyperbolic geometry used there.

Acknowledgments. This article is prepared for the proceedings of the conference "Knots in Poland 2003" held at the Stefan Banach International Mathematical Center from 7th to 27th July, 2003. I would like to thank the organizers and the Banach Center for their hospitality.

Thanks are also due to K. Masuda, who helped me in drawing Figures 2026 by using Mathematica.

2. Main results. Let $E$ be the figure-eight knot $4_{1}$. According to K. Habiro and T. Le, $J_{N}(E ; t)$ can be given as follows [4] (see also [11]):

$$
J_{N}(E ; t)=\sum_{l=0}^{N-1} \prod_{k=1}^{l} t^{N}\left(1-t^{-N-k}\right)\left(1-t^{-N+k}\right) .
$$

It is proved in [18] that the asymptotic behavior of $J_{N}(E ; \exp (2 \pi r \sqrt{-1} / N))$ for large $N$ determines the volume and the Chern-Simons invariant of the three-manifold obtained from the three-sphere $S^{3}$ by Dehn surgery along $E$ parameterized by a complex number $r$ near 1 .

Theorem $2.1([18])$. Put

$$
H(z, w):=\operatorname{Li}_{2}\left(z^{-1} w^{-1}\right)-\operatorname{Li}_{2}\left(z w^{-1}\right)+\log z \log w
$$


with

$$
\operatorname{Li}_{2}(z):=-\int_{0}^{z} \frac{\log (1-w)}{w} d z
$$

the dilogarithm function. Then there exists a neighborhood $U$ of $1 \in \mathbb{C}$ such that if $r \in(U \backslash \mathbb{Q}) \cup\{1\}$ then

$$
2 \pi r \sqrt{-1} \lim _{N \rightarrow \infty} \frac{\log J(E ; \exp (2 \pi r \sqrt{-1} / N))}{N}=H(y, \exp (2 \pi r \sqrt{-1})),
$$

where $y$ is the solution to the equation

$$
y+y^{-1}=\exp (2 \pi r \sqrt{-1})+\exp (-2 \pi r \sqrt{-1})-1 .
$$

Here we choose $y$ so that $y=\exp (-\pi \sqrt{-1} / 3)$ when $r=1$.

REMARK 2.2. If $r$ is rational with $5 / 6<r<1$ or $1<r<7 / 6$, it is proved that the superior limit is $H(y, \exp (2 \pi r \sqrt{-1}))$ but the inferior limit is 0 (see [15]).

Let $u$ be the complex number near 0 parameterizing hyperbolic structures of $S^{3} \backslash E$ described in [19]. (To be more precise, $u$ is the logarithm of the holonomy of the meridian of the boundary torus of the regular neighborhood of $E$. We also denote that of the longitude by $v$. See Section 5 for details.) It is known $[19,28]$ that there exists an analytic function of $u$ such that if $u$ determines a closed three-manifold $E_{u}$ after adding a geodesic $\gamma_{u}$ to complete the hyperbolic structure in $S^{3} \backslash E$, then the volume and the Chern-Simons invariant of $E_{u}$ can be defined by using the function and some geometric invariants of $\gamma_{u}$.

Putting $u:=2 \pi r \sqrt{-1}-2 \pi \sqrt{-1}$, we have the following corollary.

COROLlary 2.3. Let $E_{u}$ be the three-manifold obtained from the figureeight knot by Dehn surgery corresponding to u near 0 such that $\frac{u}{\pi \sqrt{-1}} \notin \mathbb{Q}$. Then

$$
\operatorname{Vol}\left(E_{u}\right)=\operatorname{Im} H(y, u)+\pi \operatorname{Re} u-\frac{\operatorname{Im}(u v)}{4}-\frac{\pi}{2} \operatorname{length}\left(\gamma_{u}\right),
$$

where length $\left(\gamma_{u}\right)$ is the length of $\gamma_{u}$ and $y$ is a solution to the equation $y+y^{-1}=u+u^{-1}-1$.

REMARK 2.4. The right hand side is the real part of the following complex-valued function:

$$
\frac{H(y, u)}{\sqrt{-1}}+\pi u-\frac{u v}{4 \sqrt{-1}}-\frac{\pi}{2} \varrho\left(\gamma_{u}\right)
$$

with $\operatorname{Re} \varrho\left(\gamma_{u}\right)=$ length $\left(\gamma_{u}\right)$. It can be shown that its imaginary part defines the Chern-Simons invariant [18]. 
3. Outline of the proof of Theorem 2.1. We first note that if $t=$ $\exp (2 \pi r \sqrt{-1} / N)$, then

$$
\prod_{k=1}^{l}\left(1-t^{-N \pm k}\right)=\exp \left\{\frac{N}{2 \pi} \sum_{k=1}^{l} \frac{2 \pi}{N} \log (1-\exp ( \pm 2 \pi k r \sqrt{-1} / N-2 \pi r \sqrt{-1}))\right\}
$$

which can be approximated by

$$
\exp \left\{\frac{N}{2 \pi} \int_{0}^{2 \pi l / N} \log (1-\exp ( \pm s r \sqrt{-1}-2 \pi r \sqrt{-1})) d s\right\}
$$

for large $N$, if $r$ is not a rational number. (Note that if $r$ is rational the product may vanish.) Putting $u:=\exp ( \pm s r \sqrt{-1}-2 \pi r \sqrt{-1})$, we have

$$
\begin{aligned}
\int_{0}^{2 \pi l / N} \log (1-\exp ( \pm s r & \sqrt{-1}-2 \pi r \sqrt{-1})) d s \\
& =\frac{ \pm 1}{r \sqrt{-1}} \int_{\exp (-2 \pi r \sqrt{-1})}^{\exp ( \pm 2 \pi l r \sqrt{-1} / N-2 \pi r \sqrt{-1})} \frac{\log (1-u)}{u} d u \\
& =\frac{ \pm 1}{r \sqrt{-1}}\left\{\operatorname{Li}_{2}\left(n^{-1}\right)-\operatorname{Li}_{2}\left(q^{ \pm l} n^{-1}\right)\right\}
\end{aligned}
$$

Here we put $n:=\exp (2 \pi r \sqrt{-1})$ and $q:=\exp (2 \pi r \sqrt{-1} / N)$.

Therefore for large $N$,

$$
\begin{aligned}
& J_{N}(E ; q) \sim \sum_{l=0}^{N-1} q^{N l} \exp \left\{\frac{N}{2 \pi r \sqrt{-1}}\left(\operatorname{Li}_{2}\left(n^{-1}\right)-\operatorname{Li}_{2}\left(q^{l} n^{-1}\right)\right)\right\} \\
& \quad \times \exp \left\{\frac{-N}{2 \pi r \sqrt{-1}}\left(\operatorname{Li}_{2}\left(n^{-1}\right)-\operatorname{Li}_{2}\left(q^{-l} n^{-1}\right)\right)\right\} \\
&= \sum_{l=0}^{N-1} \exp \left\{\frac{N}{2 \pi r \sqrt{-1}}\left(\operatorname{Li}_{2}\left(q^{-l} n^{-1}\right)-\operatorname{Li}_{2}\left(q^{l} n^{-1}\right)+\left(\log q^{l}\right)(\log n)\right)\right\} \\
&= \sum_{l=0}^{N-1} \exp \left\{\frac{N}{2 \pi r \sqrt{-1}} H\left(q^{l}, n\right)\right\} .
\end{aligned}
$$

Now since the residue around $\exp (2 \pi l \sqrt{-1} / N)$ of the function $1 /\left(z\left(1-z^{-N}\right)\right)$ is $1 / N$, from the residue theorem we have

$$
J_{N}(E ; q) \sim 1+\frac{N}{2 \pi \sqrt{-1}} \int_{C} \frac{1}{z\left(1-z^{-N}\right)} \exp \left\{\frac{N}{2 \pi r \sqrt{-1}} H\left(z^{r}, n\right)\right\} d z
$$




$$
\begin{aligned}
= & +\frac{N}{2 \pi \sqrt{-1}} \int_{C_{+}} \frac{1}{z} \exp \left\{\frac{N}{2 \pi r \sqrt{-1}} H\left(z^{r}, n\right)\right\} d z \\
& +\frac{N}{2 \pi \sqrt{-1}} \int_{C_{+}} \frac{1}{z\left(1-z^{-N}\right)} \exp \left\{\frac{N}{2 \pi r \sqrt{-1}}\left(H\left(z^{r}, n\right)-2 \pi \sqrt{-1} \log z^{r}\right)\right\} d z \\
& +\frac{N}{2 \pi \sqrt{-1}} \int_{C_{-}} \frac{1}{z\left(z^{N}-1\right)} \exp \left\{\frac{N}{2 \pi r \sqrt{-1}}\left(H\left(z^{r}, n\right)+2 \pi \sqrt{-1} \log z^{r}\right)\right\} d z
\end{aligned}
$$

where $C:=C_{+} \cup C_{-}$with

$$
\begin{aligned}
C_{+}:= & \{z \in \mathbb{C}|| z \mid=1+\varepsilon, \pi / N \leq \arg z \leq 2 \pi-\pi / N\} \\
& \cup\{t \exp (\pi \sqrt{-1} / N) \in \mathbb{C} \mid 1-\varepsilon \leq t \leq 1+\varepsilon\} \\
& \cup\{t \exp (-\pi \sqrt{-1} / N) \in \mathbb{C} \mid 1-\varepsilon \leq t \leq 1+\varepsilon\}, \\
C_{-}:= & \{z \in \mathbb{C}|| z \mid=1-\varepsilon, \pi / N \leq \arg z \leq 2 \pi-\pi / N\}
\end{aligned}
$$

for small $\varepsilon>0$. Then by the saddle point method we see that

$$
\int_{C_{+}} \frac{1}{z} \exp \left\{\frac{N}{2 \pi r \sqrt{-1}} H\left(z^{r}, n\right)\right\} d z \sim P(N) \exp \left\{\frac{N}{2 \pi r \sqrt{-1}} H(y, n)\right\},
$$

where $P(N)$ is a function of $N$ that grows polynomially $(N \rightarrow \infty)$ and

$$
y:=\frac{n-1+n^{-1}-\sqrt{\left(n-1+n^{-1}\right)^{2}-4}}{2} .
$$

Here the square root is chosen so that $y=\exp (-\pi \sqrt{-1} / 3)$ when $n=1$. Note that $y^{1 / r}$ is a solution to $\partial H\left(z^{r}, n\right) / \partial z=0$ since

$$
\frac{\partial H\left(z^{r}, n\right)}{\partial z}=\frac{r}{z} \log \left(n-\left(z^{r}+z^{-r}\right)+n^{-1}\right) .
$$

In fact $y^{1 / r}$ is the unique maximum of $\operatorname{Im} H\left(z^{r}, n\right)$ on $C_{+}$.

REMARK 3.1. A discrete version of (3.1) can be understood as follows. Let us consider the summation $\sum_{k=a}^{b} \frac{1}{k} e^{N f(k)}$. Suppose that Re $f(k)$ takes its unique maximum at $k=c$. Then

$$
\begin{aligned}
\mid \frac{\sum_{k=a}^{b} \frac{1}{k} e^{N f(k)}}{\frac{1}{c} e^{N f(c)} \mid \leq} & \frac{|c|}{|a|} e^{N \operatorname{Re}(f(a)-f(c))}+\cdots+\frac{|c|}{|c-1|} e^{N \operatorname{Re}(f(c-1)-f(c))}+1 \\
& +\frac{|c|}{|c+1|} e^{N \operatorname{Re}(f(c+1)-f(c))}+\cdots+\frac{|c|}{|b|} e^{N \operatorname{Re}(f(b)-f(c))} \\
& \stackrel{N \rightarrow \infty}{\longrightarrow} 1 .
\end{aligned}
$$

Therefore

$$
\sum_{k=a}^{b} \frac{1}{k} e^{N f(k)} \underset{N \rightarrow \infty}{\sim} \frac{1}{c} e^{N f(c)} .
$$


It can also be proved that

$$
\operatorname{Re}\left(\frac{H\left(z^{r}, n\right) \mp 2 \pi \sqrt{-1} \log z^{r}}{2 \pi r \sqrt{-1}}\right)<\operatorname{Re}\left(\frac{H(y, n)}{2 \pi r \sqrt{-1}}\right)
$$

for $z \in C_{ \pm}$if $r$ is near 1 . Therefore the absolute value of

$$
\int_{C_{ \pm}} \frac{ \pm 1}{z\left(1-z^{\mp N}\right)} \exp \left\{\frac{N}{2 \pi r \sqrt{-1}}\left(H\left(z^{r}, n\right) \mp 2 \pi \sqrt{-1} \log z^{r}\right)\right\} d z
$$

is strictly smaller than that of $\exp \left(\frac{N}{2 \pi r \sqrt{-1}} H(y, n)\right)$ times $Q_{ \pm}(N)$, where $Q_{ \pm}(N)$ is a function of $N$ with polynomial growth. So we finally see that

$$
\begin{aligned}
& \frac{\log \left(J_{N}(E ; q)\right)}{N}-\frac{H(y, n)}{2 \pi r \sqrt{-1}} \\
& =\log \left(\frac{J_{N}(E ; q)}{\exp \left(\frac{N}{2 \pi r \sqrt{-1}} H(y, n)\right)}\right) / N \\
& \sim \log \left(\exp \left(\frac{-N}{2 \pi r \sqrt{-1}} H(y, n)\right)+\frac{N}{2 \pi \sqrt{-1}}\left(P(N)+Q_{+}(N)+Q_{-}(N)\right)\right) / N \\
& \stackrel{N \rightarrow \infty}{\longrightarrow} 0 .
\end{aligned}
$$

4. Volume calculation. In this section I will calculate the volume of the figure-eight knot complement $S^{3} \backslash E$ with a (complete or incomplete) hyperbolic structure.

First note that one can decompose $S^{3} \backslash E$ into two hyperbolic ideal tetrahedra [22, Chapter 1], [23, Example 1.4.8]. Here an ideal tetrahedron is a tetrahedron without vertices. Let $\mathbb{H}^{3}$ be the three-dimensional hyperbolic space modeled on the upper half space $\mathbb{R}_{+}^{3}=\{(x, y, \tau) \mid x, y, \tau \in \mathbb{R}, \tau>0\}$ with metric $d s^{2}=\left(d x^{2}+d y^{2}+d \tau^{2}\right) / \tau^{2}$. Note that in $\mathbb{H}^{3}$, a "flat" plane is either a (usual Euclidean) plane perpendicular to the $x y$-plane or an upper hemisphere centered at a point on the $x y$-plane, and that a "straight" line is either a (usual Euclidean) line perpendicular to the $x y$-plane or an upper semicircle centered at a point on the $x y$-plane. The group of isometries of $\mathbb{H}^{3}$ can be regarded as $\operatorname{PSL}(2, \mathbb{C})$, where $\left(\begin{array}{ll}a & b \\ c & d\end{array}\right) \in \operatorname{PSL}(2, \mathbb{C})$ acts on $\mathbb{H}^{3}$ as follows. First $\left(\begin{array}{ll}a & b \\ c & d\end{array}\right)$ acts on $\mathbb{C}$ or rather its one-point compactification $\hat{\mathbb{C}}$ by $z \mapsto \frac{a z+b}{c z+d}$. Regarding the $x y$-plane as $\mathbb{C}$ and $\hat{\mathbb{C}}$ as the boundary of $\mathbb{H}^{3}$, the action of $\left(\begin{array}{ll}a & b \\ c & d\end{array}\right)$ on $\mathbb{H}^{3}$ is the natural (Poincaré) extension.

Now by an isometry, we may assume that any ideal hyperbolic tetrahedron can be put so that a vertex is $\infty \in \hat{\mathbb{C}}=\partial \mathbb{H}^{3}$. (Note that every vertex of an ideal hyperbolic tetrahedron is on the sphere at infinity $\hat{\mathbb{C}}$.) Then an ideal hyperbolic tetrahedron looks like Figure 1; it is bounded by three planes perpendicular to the $x y$-plane and one hemisphere. It can be 

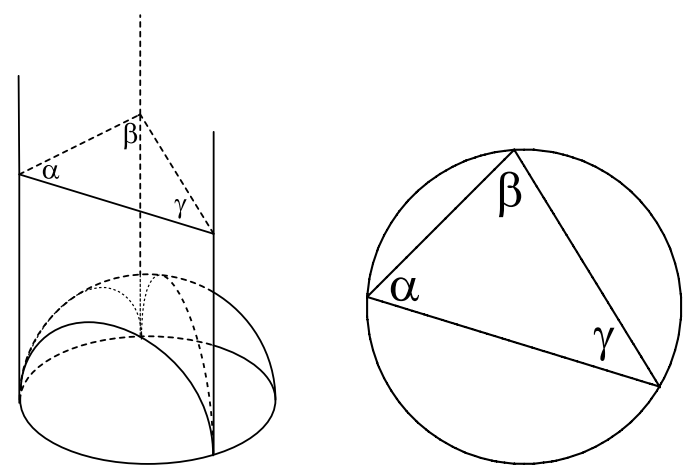

Fig. 1. An ideal hyperbolic tetrahedron.
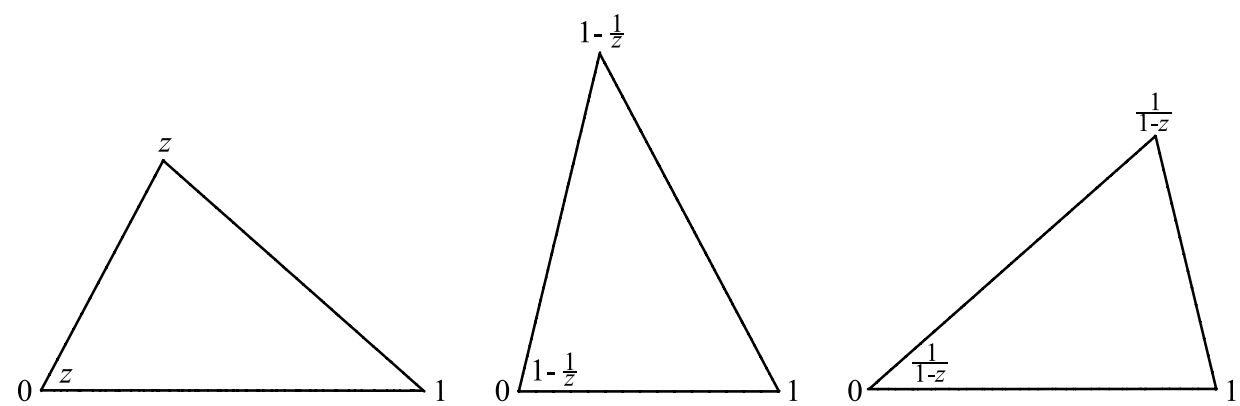

Fig. 2. Three triangles represent the same ideal hyperbolic tetrahedron.

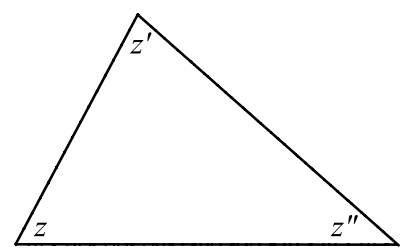

Fig. 3. Three complex numbers $z, z^{\prime}:=1-\frac{1}{z}$, and $z^{\prime \prime}:=\frac{1}{1-z}$ define the same ideal hyperbolic tetrahedron.

shown that the isometry class of an ideal hyperbolic tetrahedron is determined by the similarity class of the triangle appearing in the intersection of the three planes and a plane parallel to the $x y$-plane. Putting a triangle on $\mathbb{C}$ so that two vertices are at 0 and 1 , and the other at $z$ with $\operatorname{Im} z>0$, we see that the isometry classes of ideal hyperbolic tetrahedra are parameterized by complex numbers with positive imaginary parts. Note that there are three ways to parameterize an ideal hyperbolic tetrahedron; $z, 1-\frac{1}{z}$, and $\frac{1}{1-z}$. See Figure 2, where $\arg z=\alpha, \arg \left(1-\frac{1}{z}\right)=\beta$, and $\arg \frac{1}{1-z}=\gamma$. We associate to vertices complex numbers $z, z^{\prime}:=1-\frac{1}{z}, z^{\prime \prime}:=\frac{1}{1-z}$ as in Figure 3. We can also associate to three edges of the tetrahedron $z, z^{\prime}$, 

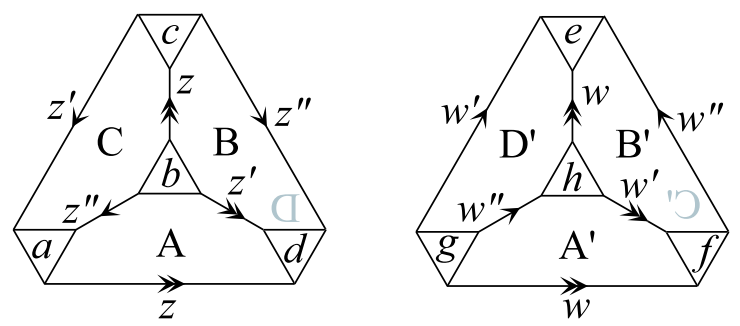

Fig. 4. Two truncated tetrahedra parameterized by $z$ and $w$. The triangles with labels $a, \ldots, h$ correspond to the removed vertices.

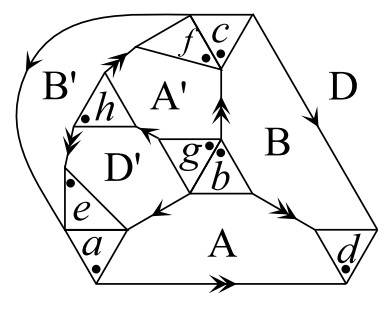

Fig. 5. Glue $\mathrm{C}$ and $\mathrm{C}^{\prime}$. The face $\mathrm{D}$ is the unbounded region.

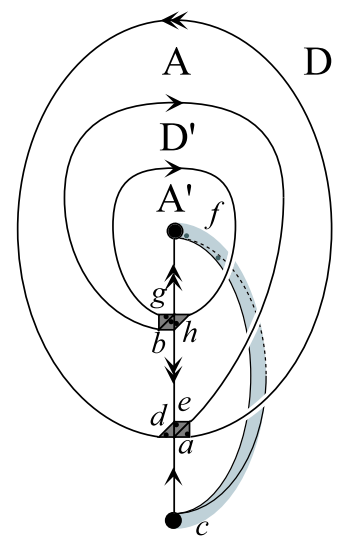

Fig. 7. Glue $\mathrm{B}$ and $\mathrm{B}^{\prime}$. The triangles $c$ and $f$ make a tube.

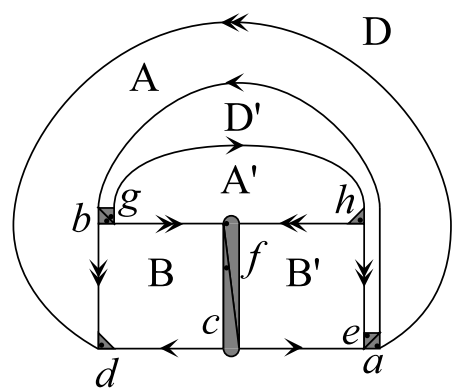

Fig. 6. Arrange Figure 5 .

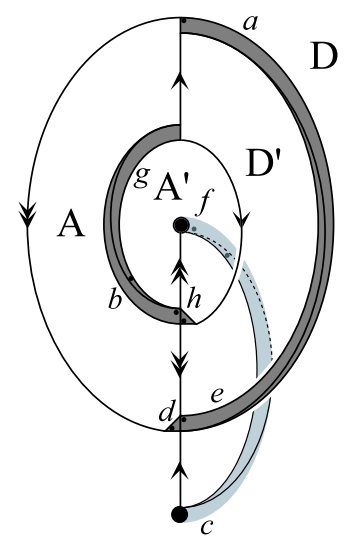

Fig. 8. Arrange Figure 7.

and $z^{\prime \prime}$. By putting other vertices at $\infty$, we associate to each edge one of $z, z^{\prime}$, or $z^{\prime \prime}$. It can be proved that opposite edges share the same complex parameter as in Figure 4. See [22, Section 4.1].

We prepare two ideal hyperbolic tetrahedra parameterized by $z$ (with faces $\mathrm{A}, \mathrm{B}, \mathrm{C}$, and $\mathrm{D}$ ) and $w$ (with faces $\mathrm{A}^{\prime}, \mathrm{B}^{\prime}, \mathrm{C}^{\prime}$, and $\mathrm{D}^{\prime}$ ) as in Figure 4 . 


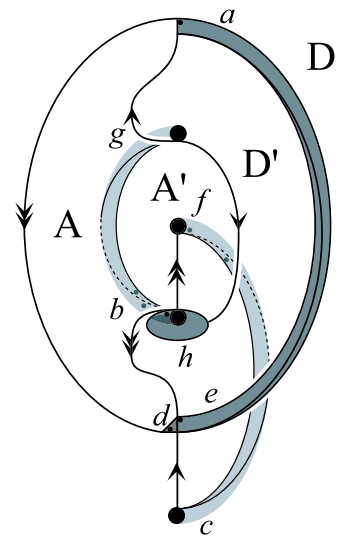

Fig. 9. Glue sides of the triangles $b$ and $g$ to make a tube.

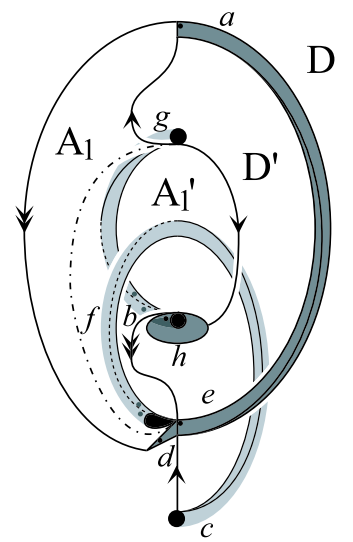

Fig. 11. Glue $\mathrm{A}_{2}$ and $\mathrm{A}_{2}^{\prime}$.

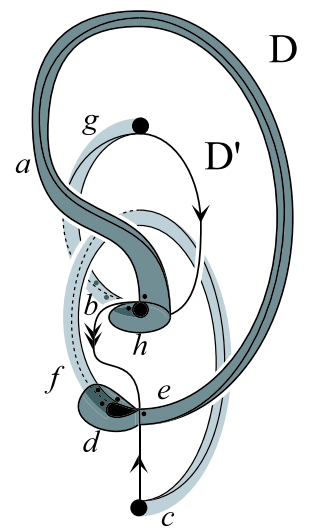

Fig. 13. Glue $A_{1}$ and $A_{1}^{\prime}$.

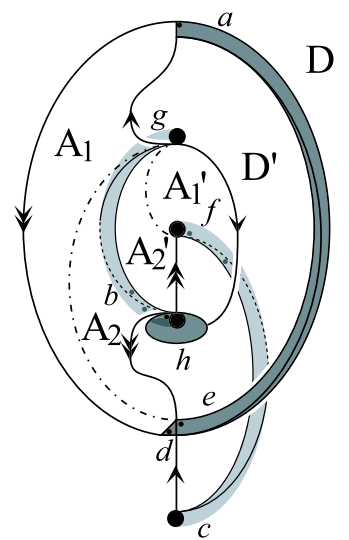

Fig. 10. Divide $A\left(\mathrm{~A}^{\prime}\right.$, respectively) into $A_{1}$ and $A_{2}\left(A_{1}^{\prime}\right.$ and $A_{2}^{\prime}$, respectively).

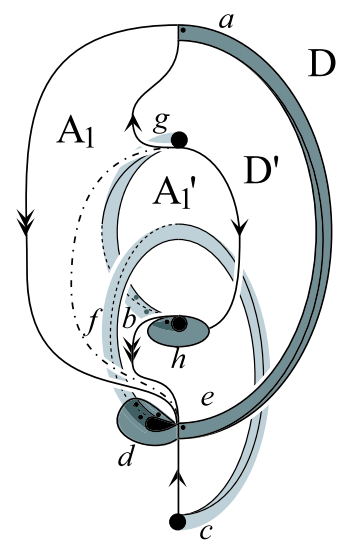

Fig. 12. Glue sides of $d$ and $f$. The regions $\mathrm{A}_{1}$ and $\mathrm{A}_{1}^{\prime}$ become smaller.

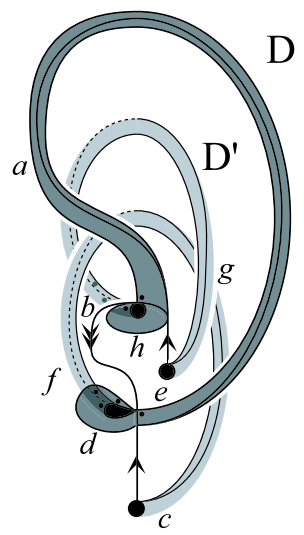

Fig. 14. Arrange Figure 13. 


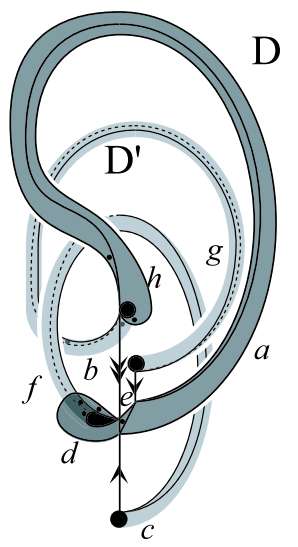

Fig. 15. Arrange Figure 14.

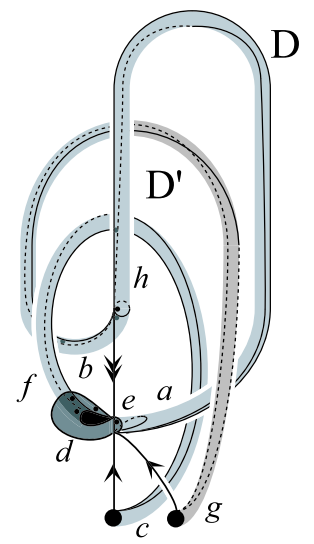

Fig. 17. Arrange Figure 16.

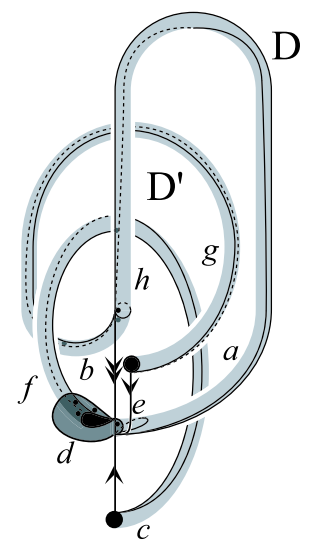

Fig. 16. Glue sides of $a$ and $h$ to make a tube.

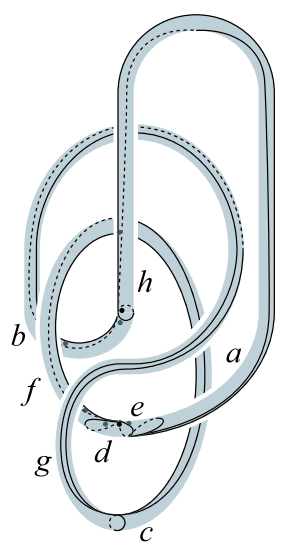

Fig. 18. Glue $\mathrm{D}$ and $\mathrm{D}^{\prime}$. The resulting torus is the boundary of the regular neighborhood of the figure-eight knot.

We consider the corresponding truncated tetrahedra, where triangles labeled with $a, \ldots, h$ appear. Then glue them so that $\mathrm{A}$ is identified with $\mathrm{A}^{\prime}$, and so on. See Figures 5-18 to see this gives the figure-eight knot complement. In these figures dots stand for the vertices corresponding to $z$ or $w$.

Now the eight triangles corresponding to the eight vertices of the tetrahedra make a torus (cusp torus) as in Figure 19.

Since the product of the parameters corresponding to the angles around a vertex, which correspond to the dihedral angles around an edge in the tetrahedron decomposition of $S^{3} \backslash E$, should be 1, we have the following equations:

$$
z^{\prime}\left(z^{\prime \prime}\right)^{2} w^{\prime}\left(w^{\prime \prime}\right)^{2}=1 \quad \text { and } \quad z^{2} z^{\prime} w^{2} w^{\prime}=1,
$$




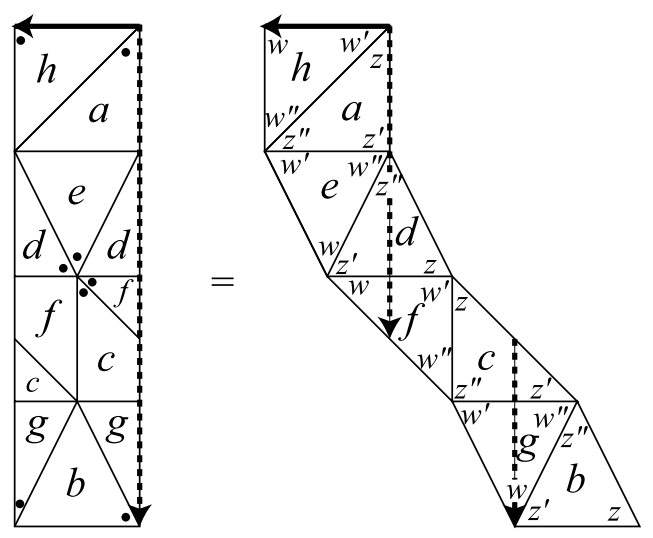

Fig. 19. The torus corresponding to the boundary of the regular neighborhood of the figure-eight knot, where the thick line indicates the meridian and the dotted thick line indicates the longitude. Note that here we are watching the torus from outside the figure-eight knot complement.

which is equivalent to the single equation

$$
z^{\prime} w^{\prime}=\left(1-z^{\prime}\right)^{2}\left(1-w^{\prime}\right)^{2}
$$

Let us introduce a parameter $n:=w(1-z)$. Then $z^{\prime}$ and $w^{\prime}$ satisfy the equalities

$$
\frac{z^{\prime}}{n}+\frac{n}{z^{\prime}}=n-1+\frac{1}{n}, \quad n w^{\prime}+\frac{1}{n w^{\prime}}=n-1+\frac{1}{n} .
$$

We put $y:=n / z^{\prime}=1 /\left(n w^{\prime}\right)$. Then $y$ satisfies

$$
y+\frac{1}{y}=n-1+\frac{1}{n} .
$$

Note that $y, z, w$ (and so $z^{\prime}, z^{\prime \prime}, w^{\prime}$, and $w^{\prime \prime}$ ) are all functions of $n$.

We will calculate the volume $\operatorname{Vol}(n)$ of the figure-eight knot complement corresponding to the parameter $n$. It is well known (see for example [22, Theorem 7.2.1]) that the volume of the ideal hyperbolic tetrahedron parameterized by $z$ is $\pi(\arg z)+Л\left(\arg z^{\prime}\right)+\pi\left(\arg z^{\prime \prime}\right)$, where $\Pi$ is the Lobachevsky function

$$
\pi(\theta):=-\int_{0}^{\theta} \log |2 \sin \varphi| d \varphi .
$$

Therefore we have

$\operatorname{Vol}(n)=\pi(\arg z)+Л\left(\arg z^{\prime}\right)+Л\left(\arg z^{\prime \prime}\right)+Л(\arg w)+Л\left(\arg w^{\prime}\right)+Л\left(\arg w^{\prime \prime}\right)$.

We want to express the right hand side in terms of $n$ and $y$ using log and $\mathrm{Li}_{2}$. (Note that $y$ is determined by $n$.)

We use the following two identities:

$$
\operatorname{Im} \operatorname{Li}_{2}(\zeta)=\pi(\arg \zeta)+\pi\left(\arg \zeta^{\prime}\right)+\pi\left(\arg \zeta^{\prime \prime}\right)-\log |\zeta| \arg (1-\zeta)
$$

for $\operatorname{Im} \zeta>0$ (see for example [19, p. 324]), and

$$
\mathrm{Li}_{2}(\zeta)+\mathrm{Li}_{2}\left(\zeta^{-1}\right)+\frac{\pi^{2}}{6}+\frac{1}{2}(\log (-\zeta))^{2}=0
$$


for $\zeta \in \mathbb{C} \backslash[1, \infty)$ (see for example [29, p. 134]). Here the branch of log is assumed to be so that $\log 1=0$. Therefore

$$
\begin{aligned}
& \operatorname{Vol}(n)=\operatorname{ImLi}_{2}\left(z^{\prime}\right)+\log \left|z^{\prime}\right| \arg \left(1-z^{\prime}\right)+\operatorname{Im}_{L_{2}}\left(w^{\prime}\right)+\log \left|w^{\prime}\right| \arg \left(1-w^{\prime}\right) \\
& =\operatorname{Im} \operatorname{Li}_{2}\left(w^{\prime}\right)+\log \left|w^{\prime}\right| \arg \left(1-w^{\prime}\right)-\operatorname{ImLi}_{2}\left(z^{\prime-1}\right) \\
& -\log \left|z^{\prime-1}\right| \arg \left(1-z^{\prime-1}\right) \\
& =\operatorname{ImLi} \operatorname{Li}_{2}\left(y^{-1} n^{-1}\right)-\operatorname{ImLi}_{2}\left(y n^{-1}\right) \\
& +\log \left|y^{-1} n^{-1}\right| \arg \left(1-y^{-1} n^{-1}\right)-\log \left|y n^{-1}\right| \arg \left(1-y n^{-1}\right) \\
& =\operatorname{Im} \operatorname{Li}_{2}\left(y^{-1} n^{-1}\right)-\operatorname{ImLi}_{2}\left(y n^{-1}\right) \\
& -\log |y| \arg \left(1-y^{-1} n^{-1}\right)\left(1-y n^{-1}\right)+\log |n| \arg \frac{1-y n^{-1}}{1-y^{-1} n^{-1}} \\
& =\operatorname{Im} \operatorname{Li}_{2}\left(y^{-1} n^{-1}\right)-\operatorname{Im} \operatorname{Li}_{2}\left(y n^{-1}\right) \\
& -\log |y| \arg \left(n^{-1}\left(n+n^{-1}-y-y^{-1}\right)\right)+\log |n| \arg \frac{1-y n^{-1}}{1-y^{-1} n^{-1}} \\
& =\operatorname{ImLi}_{2}\left(y^{-1} n^{-1}\right)-\operatorname{ImLi}_{2}\left(y n^{-1}\right)+\log |y| \arg n \\
& +\log |n| \arg \frac{1-y n^{-1}}{1-y^{-1} n^{-1}} .
\end{aligned}
$$

Since $n$ is near 1 , and $y$ is near $\exp (-\pi \sqrt{-1} / 3)$, we have

$$
\arg \frac{1-y n^{-1}}{1-y^{-1} n^{-1}}= \begin{cases}\arg y-\arg \frac{y-n^{-1}}{1-y n^{-1}} & \text { if } \arg \frac{y-n^{-1}}{1-y n^{-1}}<0, \\ \arg y-\arg \frac{y-n^{-1}}{1-y n^{-1}}+2 \pi & \text { if } \arg \frac{y-n^{-1}}{1-y n^{-1}}>0 .\end{cases}
$$

We also have

$$
\frac{y-n^{-1}}{1-y n^{-1}}=z(z-1)
$$

since

$$
\left(y-n^{-1}\right)\left(1-y n^{-1}\right)=y n^{-1} .
$$

Putting

$$
\left\{\begin{array}{l}
u:=\log n \\
v:=2 \log (z(1-z))=2 \log \frac{-y n^{-1}}{\left(1-y n^{-1}\right)^{2}},
\end{array}\right.
$$

we have

$$
\begin{aligned}
& \operatorname{Vol}(n) \\
& = \begin{cases}\operatorname{Im} H(y, n)-\log |n| \arg (z(z-1)), & \arg (z(z-1))<0, \\
\operatorname{Im} H(y, n)-\log |n|(\arg (z(z-1))-2 \pi), & \arg (z(z-1))>0,\end{cases}
\end{aligned}
$$




$$
\begin{aligned}
& = \begin{cases}\operatorname{Im} H(y, n)-\log |n|(\arg (z(1-z))-\pi), & \arg (z(z-1))<0, \\
\operatorname{Im} H(y, n)-\log |n|(\arg (z(1-z))+\pi-2 \pi), & \arg (z(z-1))>0,\end{cases} \\
& =\operatorname{Im} H(y, n)-\log |n|(\arg (z(1-z))-\pi) \\
& =\operatorname{Im} H(y, n)+\pi \operatorname{Re} u-\frac{1}{2} \operatorname{Re} u \operatorname{Im} v .
\end{aligned}
$$

5. Geometric interpretation of the volume formula. In this section I will prove Corollary 2.3.

First I describe a geometric interpretation of $u$ and $v$ appearing in (4.1). See $[1,22]$ for details.

Let $K$ be a hyperbolic knot in $S^{3}$, that is, $S^{3} \backslash K$ has a complete hyperbolic structure. We assume that the complement $S^{3} \backslash K$ is decomposed into hyperbolic ideal tetrahedra. Note that all the (removed) vertices of these tetrahedra are identified to the "point at infinity" $X$. Since the regular neighborhood of each ideal vertex is homeomorphic to (a triangle) $\times[0, \infty)$ ( $\infty$ corresponds to the vertex), the regular neighborhood $\mathcal{E}$ of $X$ is homeomorphic to $T \times[0, \infty)$ with $T$ a torus. We call $\mathcal{E}$ the end of $S^{3} \backslash E$ and $T$ the cusp torus. The torus $T$ has a Euclidean structure coming from the complete hyperbolic structure in $S^{3} \backslash K$. We orient $T$ as in Figure 19, that is, by using the meridian-longitude pair $(\mu, \lambda)$. If we change the hyperbolic structure slightly, we get an incomplete hyperbolic structure in $S^{3} \backslash K$ and a similarity structure on $T$.

Let $D: \tilde{T} \rightarrow \mathbb{C}$ be the developing map, where $\tilde{T}$ is the universal cover of $T$, which is homeomorphic to $\mathbb{R}^{2}$. Geometrically $D$ can be described as follows. Note that $T$ is decomposed into triangles each of which is determined up to similarity. Cut $T$ into a quadrilateral $S$ consisting of triangles. Map each triangle in $S$ into $\mathbb{C}$ preserving the similarity structure so that the edges fit in $\mathbb{C}$. Then the image $D(S)$ of $S$ is defined as a polygon with four sides corresponding to the four sides of $S$. Noting that each pair of opposite sides can be identified by a similarity transformation, we can paste copies of $D(S)$ side by side on $\mathbb{C}$, defining $D: \tilde{T} \rightarrow \mathbb{C}$.

Example 5.1. Let us consider the case when $K$ is the figure-eight knot. Figures 20-23 show an example when the translations of $D(S)$ give a tiling of $\mathbb{C} \backslash\{0\}$. Figures 24 and 25 show another example of such a case. Figure 26 shows an example when translations of $D(S)$ are dense.

We also denote by $D: \tilde{\mathcal{E}} \rightarrow \mathbb{H}^{3}$ the developing map from the universal cover of $\mathcal{E}$, which is homeomorphic to $\mathbb{R}^{2} \times[0, \infty)$, where the point at infinity $X$ is sent to $\infty$.

We assume that $T$ has a similarity structure. Let $\operatorname{Sim}\left(\mathbb{R}^{2}\right)$ be the group of similarity transformations of $\mathbb{R}^{2}$, which is equal to the group of affine 


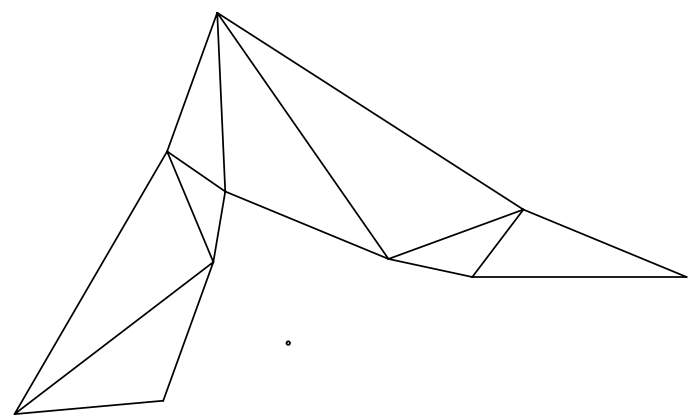

Fig. 20. $D(S)$ of the $(3,-2)$-Dehn surgery along the figure-eight knot. Here a dot indicates $0 \in \mathbb{C}$.

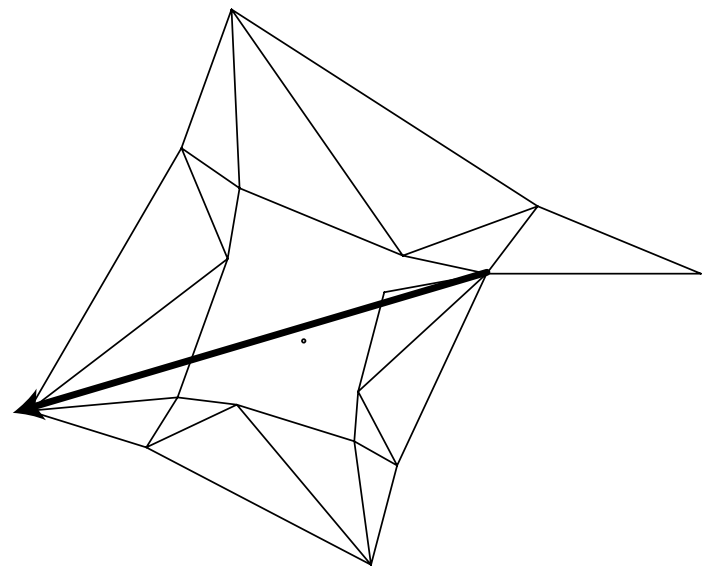

Fig. 21. The arrow indicates $\delta(\mu) \delta(\lambda)^{-1}$. Note that $\delta(\mu)^{3} \delta(\lambda)^{-2}$ is the rotation by $2 \pi$.

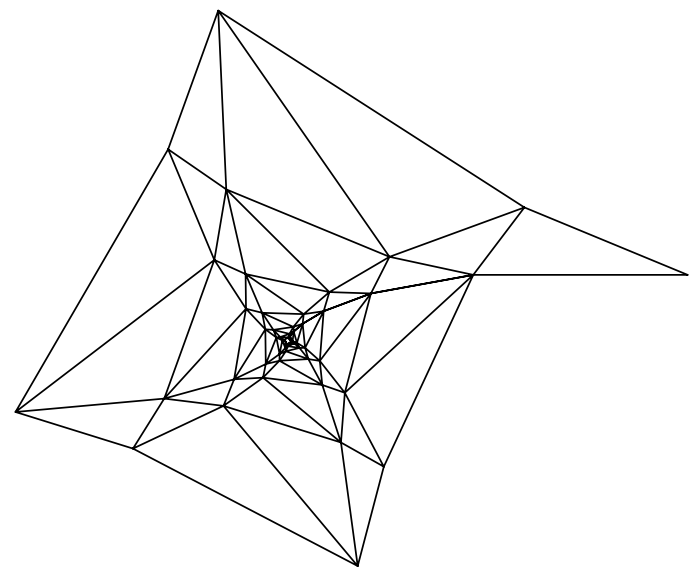

Fig. 22. Copies of $D(S)$ define a tiling of $\mathbb{C}^{*}$. 


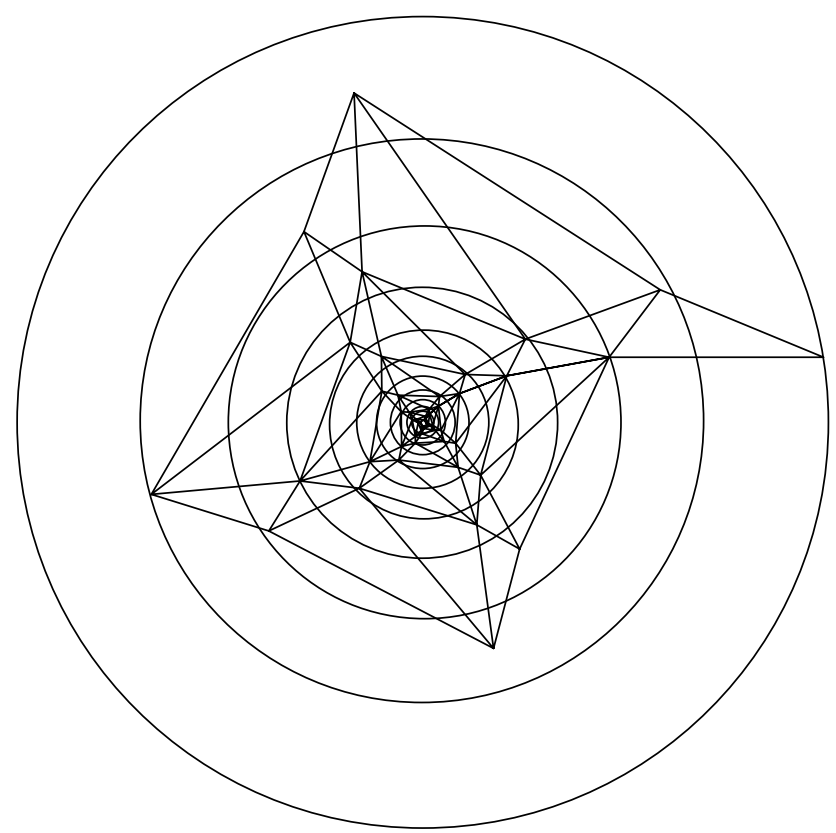

Fig. 23. Fundamental domains of Figure 22.

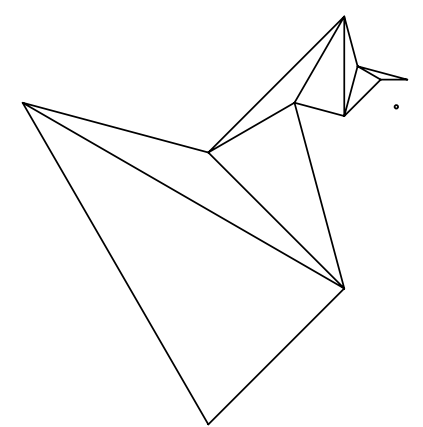

Fig. 24. $D(S)$ of the $(6,0)$-Dehn surgery along the figure-eight knot. A dot indicates $0 \in \mathbb{C}$.

transformations of $\mathbb{C}$. Then the holonomy $H: \pi_{1}(T) \rightarrow \operatorname{Sim}\left(\mathbb{R}^{2}\right)$ is defined as follows. An element $\eta \in \pi_{1}(T)$ acts on $\tilde{T}$ as a covering transformation $\sigma_{\eta}$. We define $H(\eta)$ so that $D \circ \sigma_{\eta}=H(\eta) \circ D$ holds. By changing the developing map by a translation if necessary, we may assume that $H(\eta)$ fixes $\{0, \infty\} \in \mathbb{C}$ for any $\eta \in \pi_{1}(T)$ since $\pi_{1}(T)$ is abelian. Then $H(\eta)$ acts on $\mathbb{C}$ as

$$
H(\eta)(z)=\delta(\eta) z
$$

Note that $\delta: \pi_{1}(T) \rightarrow \mathbb{C}^{*}(:=\mathbb{C} \backslash\{0\})$ is a homomorphism. 


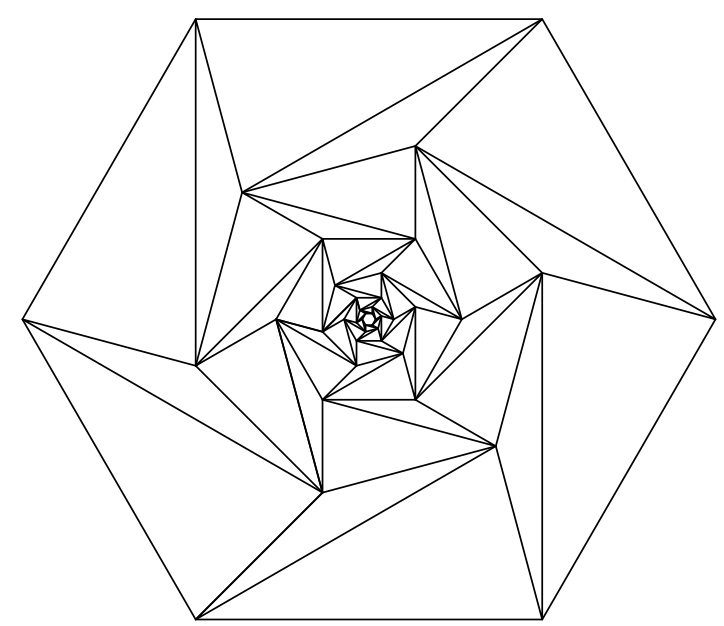

Fig. 25. Copies of $D(S)$ for the $(6,0)$-Dehn surgery along the figure-eight knot.

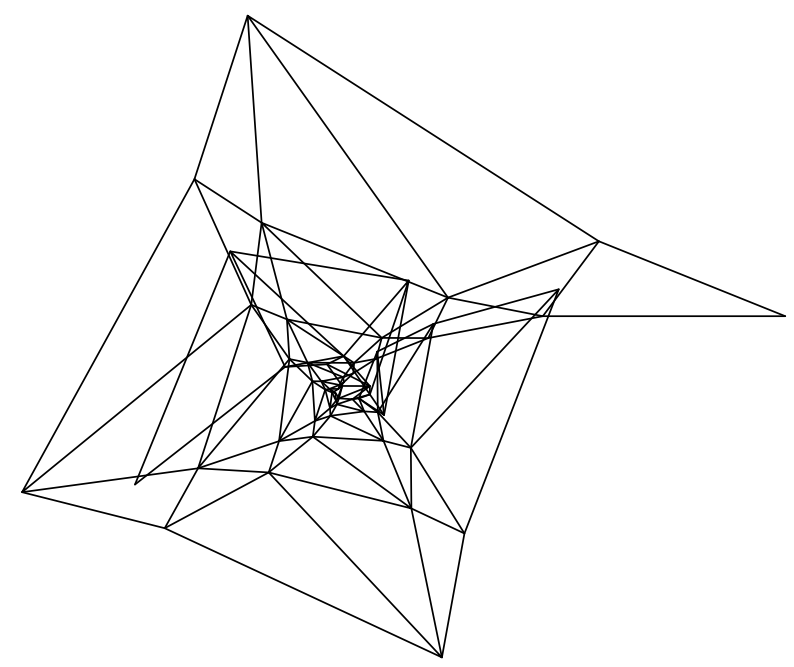

Fig. 26. Copies of $D(S)$ corresponding to irrational $p / q$.

The Poincaré extension of the holonomy $H(\eta)$ to $\mathbb{H}^{3}$ is given by

$$
H(\eta)(z, \tau)=(\delta(\eta) z,|\delta(\eta)| \tau) .
$$

Therefore we can choose $\mathcal{E}$ so that the image of the developing map $D(\tilde{\mathcal{E}})$ is a cone $C$ centered at the $\tau$-axis minus the axis:

$$
D(\tilde{\mathcal{E}})=C:=\{(z, \tau)|\tau \geq c| z \mid, z \neq 0\} \quad(c>0) .
$$

EXAmple 5.2. We describe $\delta$ in the case of the figure-eight knot. The meridian $\mu$ and the longitude $\lambda$ are indicated by the solid arrow and the 
dotted arrow respectively in Figure 19. Note that we view the torus from the outside of the knot complement.

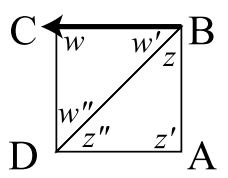

Fig. 27. The meridian $\mu$ is indicated by the arrow.

Then $\delta(\mu)$ can be read as follows. In Figure 27, multiplying $\overrightarrow{B A}$ by $z^{-1}$ we get $\overrightarrow{B D}$ and multiplying $\overrightarrow{B D}$ by $w^{\prime \prime}$ we get $\overrightarrow{C D}$. Since $\delta(\mu)$ takes $\overrightarrow{B A}$ to $\overrightarrow{C D}$

$$
\delta(\mu)=z^{-1} w^{\prime \prime}=z^{-1}(1-w)^{-1}=w(1-z) .
$$

Similarly from Figure 19, the longitude is the arrow along the right side of the left picture, which equals the right side of the right picture and twice the meridian. Therefore

$$
\begin{aligned}
\delta(\lambda) & =w^{\prime}\left(z^{\prime \prime}\right)^{-1} w^{\prime \prime}\left(z^{\prime}\right)^{-1} w^{\prime}\left(z^{\prime \prime}\right)^{-1} w^{\prime \prime}\left(z^{\prime}\right)^{-1} \times \delta(\mu)^{2} \\
& =\left(w^{\prime}\right)^{2}\left(w^{\prime \prime}\right)^{2}\left(z^{\prime}\right)^{-2}\left(z^{\prime \prime}\right)^{-2} w^{2}(1-z)^{2} \\
& =\frac{\left(1-w^{-1}\right)^{2}(1-z)^{4} w^{2}}{(1-w)^{2}\left(1-z^{-1}\right)^{2}}=z^{2}(1-z)^{2} .
\end{aligned}
$$

Hence we have

$$
u=\log \delta(\mu), \quad v=\log \delta(\lambda) .
$$

We will study the completion $\overline{\mathcal{E}}$ of $\mathcal{E}$. Note that since $D(\tilde{\mathcal{E}})$ is $C$, a cone minus the axis, its completion $\overline{D(\tilde{\mathcal{E}})}$ is the whole cone $\bar{C}$.

As in the case of the figure-eight knot (Example 5.2) we put

$$
u:=\log \delta(\mu), \quad v:=\log \delta(\lambda) .
$$

Let $p$ and $q$ be the unique real solution to the equation

$$
p u+q v=2 \pi \sqrt{-1} .
$$

Note that it is equivalent to the following system of equations:

$$
\left\{\begin{array}{l}
p \operatorname{Re} u+q \operatorname{Re} v=0, \\
p \operatorname{Im} u+q \operatorname{Im} v=2 \pi .
\end{array}\right.
$$

Suppose first that $p / q$ is irrational. In this case the set

$$
\left\{|\delta(\mu)|^{a}|\delta(\lambda)|^{b} \mid a, b \in \mathbb{Z}\right\}
$$

is dense in $\mathbb{R}_{+}:=\{\tau \in \mathbb{R} \mid \tau>0\}$, since $a \operatorname{Re} u+b \operatorname{Re} v$ can be arbitrarily close to 0 . Considering the geodesic in $\mathbb{H}^{3}$ starting at the point $\delta(\mu)^{a} \delta(\lambda)^{b} \in$ 
$\mathbb{C}^{*}$ and going along the semicircle centered at 0 and perpendicular to the $x y$-plane, we can associate each point in

$$
\left\{\delta(\mu)^{a} \delta(\lambda)^{b} \mid a, b \in \mathbb{Z}\right\}
$$

with a limit point on the $\tau$-axis. If we regard the lattice (5.2) as the vertices of "quadrilaterals", copies of $D(S)$ s, the points in (5.2) are identified in $T$ and the associated limit points (5.1) are dense in $\mathbb{R}_{+}$. So we see that $\overline{\mathcal{E}}$ is the one-point completion of $\mathcal{E}$. Note that in this case the completion of $S^{3} \backslash K$ is not a manifold. See Figure 26 for the figure-eight knot case.

Next we assume that $p / q$ is rational. Let $p^{\prime}$ and $q^{\prime}$ be coprime integers such that $p / q=p^{\prime} / q^{\prime}$. Then there exist integers $s$ and $t$ satisfying $t p^{\prime}-$ $s q^{\prime}=-1$. Now we see that the subgroup (additively) generated by $u$ and $v$ is the same as the subgroup generated by $p^{\prime} u+q^{\prime} v$ and $s u+t v$. Putting $d=p / p^{\prime}=q / q^{\prime}$, we have

$$
p^{\prime} u+q^{\prime} v=\frac{p u+q v}{d}=\frac{2 \pi \sqrt{-1}}{d} .
$$

So Image $\delta$ is generated by $\delta(\mu)^{s} \delta(\lambda)^{t}$ and the $2 \pi / d$-rotation.

Therefore we can assume that a "fundamental domain" of the developing image of the cusp torus is a sector of an annulus between the circles centered at the origin with radii 1 and $|\exp (s u+t v)|$, and with central angle $2 \pi / d$.

Let us consider examples for the figure-eight knot case.

Example 5.3. (1) Consider the case $(p, q)=(3,-2)$ (Figures 20-23). In this case $(s, t)=(1,-1)$ and so Image $(\log \delta)$ is generated by $3 u-2 v$ and $u-v$. We calculate by using Mathematica $u=-0.723043 \ldots+\sqrt{-1} \cdot 0.179545 \ldots$. and $v=-1.08456 \ldots-\sqrt{-1} \cdot 2.87228 \ldots$ Therefore $3 u-2 v=2 \pi \sqrt{-1}$ and $u-v=0.361522 \ldots+\sqrt{-1} \cdot 3.05182 \ldots$ and so Image $\delta$ is generated by $\exp (u-v)=-1.42973 \ldots+\sqrt{-1} \cdot 0.128696 \ldots$ Note that $|\exp (u-v)|=$ $1.43551 \ldots$ and $\arg \exp (u-v)=2 \pi \cdot 0.485712 \ldots$

(2) Let $(p, q)=(6,0)$ (Figures 24 and 25). Then $u=2 \pi \sqrt{-1} / 6$ and $v=$ $-\log (7+4 \sqrt{3})$. Therefore Image $\delta$ is generated by enlargement by $7+4 \sqrt{3}$ and rotation by $2 \pi / 6$.

We will calculate $|\exp (s u+t v)|=\exp (s \operatorname{Re} u+t \operatorname{Re} v)$. Since

$$
\left(\begin{array}{l}
p \\
q
\end{array}\right)=\frac{1}{\operatorname{Re} u \operatorname{Im} v-\operatorname{Im} u \operatorname{Re} v}\left(\begin{array}{rr}
\operatorname{Im} v & -\operatorname{Re} v \\
-\operatorname{Im} u & \operatorname{Re} u
\end{array}\right)\left(\begin{array}{c}
0 \\
2 \pi
\end{array}\right),
$$

we have

$$
\begin{aligned}
s \operatorname{Re} u+t \operatorname{Re} v & =\frac{s}{p^{\prime}}\left(p^{\prime} \operatorname{Re} u+q^{\prime} \operatorname{Re} v\right)-\frac{\operatorname{Re} v}{p^{\prime}}=-\frac{d \operatorname{Re} v}{p} \\
& =\frac{d(\operatorname{Re} u \operatorname{Im} v-\operatorname{Im} u \operatorname{Re} v)}{2 \pi}=-\frac{d \operatorname{Im}(u \bar{v})}{2 \pi} .
\end{aligned}
$$


REMARK 5.4. We have chosen the signs of $s$ and $t$ as above since $-\operatorname{Im} u \bar{v}$ $>0$. The reason is as follows.

By a translation we assume that $H(\mu)$ and $H(\lambda)$ fix two points $\zeta$ and $\infty$ in $\hat{\mathbb{C}}$, that is,

$$
\left\{\begin{array}{l}
H(\mu): \quad z \mapsto \delta(\mu) z+\zeta(1-\delta(\mu)), \\
H(\lambda): \quad z \mapsto \delta(\lambda) z+\zeta(1-\delta(\lambda)) .
\end{array}\right.
$$

Therefore the vertices of the developing image of a fundamental "quadrilateral" are $\mathrm{S}:=1, \mathrm{~T}:=\delta(\mu)+\zeta(1-\delta(\mu)), \mathrm{U}:=\delta(\mu) \delta(\lambda)+\zeta(1-\delta(\mu) \delta(\lambda))$, and $\mathrm{V}:=\delta(\lambda)+\zeta(1-\delta(\lambda))$. Now we consider the limit when the similarity structure goes to a Euclidean structure, that is, both $\delta(\mu)$ and $\delta(\lambda)$ go to 1 . Since the element of $\operatorname{PSL}(2, \mathbb{C})$ corresponding to a Euclidean structure has only one fixed point in $\hat{\mathbb{C}}$, we also take the limit $\zeta \rightarrow \infty$ so that the fixed point is $\infty$. Then

$$
\frac{\overrightarrow{\mathrm{SV}}}{\overrightarrow{\mathrm{ST}}}=\frac{\delta(\lambda)+\zeta(1-\delta(\lambda))}{\delta(\mu)+\zeta(1-\delta(\mu))} \stackrel{\zeta \rightarrow \infty}{\longrightarrow} \frac{1-\delta(\lambda)}{1-\delta(\mu)} \stackrel{\delta(\mu) \rightarrow 1, \delta(\lambda) \rightarrow 1}{\longrightarrow} \frac{\log \delta(\lambda)}{\log \delta(\mu)}=\frac{v}{u} .
$$

Let $\mathrm{S}, \mathrm{T}, \mathrm{U}$, and $\mathrm{V}$ approach $\mathrm{S}_{0}, \mathrm{~T}_{0}, \mathrm{U}_{0}$, and $\mathrm{V}_{0}$ respectively. Since we have chosen the orientation of the cusp torus so that $\overrightarrow{\mathrm{S}_{0} \mathrm{~V}_{0}} / \overrightarrow{\mathrm{S}_{0} \mathrm{~T}_{0}}$ is represented by a complex number with positive imaginary part, we have

$$
\operatorname{Im} u \bar{v}=\operatorname{Im} \frac{u}{v}|v|^{2}<0 .
$$

Now we consider the completion of $\mathcal{E}$. We again assume that $H(\eta)(\eta \in$ $\left.\pi_{1}(T)\right)$ acts on $\mathbb{C}^{*}$ as $z \mapsto \delta(\eta) z\left(z \in \mathbb{C}^{*}\right)$. Then this action can be extended to $\mathbb{H}^{3} \backslash \tau$-axis $=\mathbb{C}^{*} \times \mathbb{R}_{+}$as $H(\eta):(z, \tau) \mapsto(\delta(\eta) z,|\delta(\eta)| \tau)$. Therefore we have

$$
\left\{\begin{array}{l}
H\left(\mu^{p^{\prime}} \lambda^{q^{\prime}}\right):(z, \tau) \mapsto\left(\exp \left(\frac{2 \pi \sqrt{-1}}{d}\right) z, \tau\right), \\
H\left(\mu^{s} \lambda^{t}\right): \quad(z, \tau) \mapsto\left(\delta(\mu)^{s} \delta(\lambda)^{t} z, \exp \left(\frac{-d \operatorname{Im}(u \bar{v})}{2 \pi}\right) \tau\right) .
\end{array}\right.
$$

If $d=1$, then we may assume that a "fundamental domain" for $\mathcal{E}$ is the truncated cone minus the $\tau$-axis:

$$
\left\{(z, \tau)\left|1 \leq \tau \leq \exp \left(\frac{-\operatorname{Im}(u \bar{v})}{2 \pi}\right), \tau \geq c\right| z \mid, z \neq 0\right\} .
$$

Then the completion $\overline{\mathcal{E}}$ is just the whole truncated cone with top and bottom identified. Topologically it is homeomorphic to a solid torus $D^{2} \times S^{1}$. The added axis becomes a loop $\gamma_{u}$ with length

$$
\int_{1}^{\exp \left(-\frac{1}{2 \pi} \operatorname{Im}(u \bar{v})\right)} \frac{d \tau}{\tau}=-\frac{1}{2 \pi} \operatorname{Im}(u \bar{v})
$$


Therefore $S^{3} \backslash E$ with incomplete hyperbolic structure defined by $u=\exp n$ is completed by adding a geodesic loop $\gamma_{u}$ with length $-\frac{1}{2 \pi} \operatorname{Im}(u \bar{v})$. This is nothing but the $(p, q)$-Dehn surgery of $S^{3}$ along the knot $K$, since the loop represented by $p u+q v$ becomes null-homotopic.

So if $d=1$, we have

$$
\begin{aligned}
\operatorname{Vol}(n) & =\operatorname{Im} H(y, n)+\pi \operatorname{Re} u-\frac{1}{2} \operatorname{Re} u \operatorname{Im} v \\
& =\operatorname{Im} H(y, n)+\pi \operatorname{Re} u-\frac{1}{4} \operatorname{Im}(u v)-\frac{\pi}{2} \operatorname{length}\left(\gamma_{u}\right) .
\end{aligned}
$$

Recalling that $n=\exp (2 \pi r \sqrt{-1})$ and $u=\log n$, this completes the proof of Corollary 2.3. (Recall also that we take the branch of $\log$ so that $\log n=0$ when $n=1$.)

REMARK 5.5. If $d>1$, a "fundamental domain" for $\mathcal{E}$ is a sector of the truncated cone minus the $\tau$-axis:

$$
\left\{(z, \tau)\left|1 \leq \tau \leq \exp \left(\frac{-d}{2 \pi} \operatorname{Im}(u \bar{v})\right), \tau \geq c\right| z \mid, z \neq 0,0 \leq \arg z \leq \frac{2 \pi}{d}\right\} .
$$

So the completion $\overline{\mathcal{E}}$ is topologically a solid torus, but geometrically it has a singularity along the added loop, called cone-singularity with cone-angle $2 \pi / d$.

\section{References}

[1] R. Benedetti and C. Petronio, Lectures on Hyperbolic Geometry, Universitext, Springer, Berlin, 1992.

[2] M. Gromov, Volume and bounded cohomology, Inst. Hautes Études Sci. Publ. Math. 56 (1982), 5-99.

[3] S. Gukov, Three-dimensional quantum gravity, Chern-Simons theory, and the Apolynomial, HUTP-03/A003, ITEP-TH-50/02, arXiv:hep-th/0306165.

[4] K. Habiro, On the colored Jones polynomials of some simple links, Sūrikaisekikenkyūsho Kōkyūroku 1172 (2000), 34-43.

[5] K. Hikami, Quantum invariant for torus link and modular forms, Comm. Math. Phys. 246 (2004), 403-426.

[6] K. Hikami and A. N. Kirillov, Torus knot and minimal model, Phys. Lett. B 575 (2003), 343-348.

[7] R. M. Kashaev, A link invariant from quantum dilogarithm, Modern Phys. Lett. A 10 (1995), 1409-1418.

[8] -, The hyperbolic volume of knots from the quantum dilogarithm, Lett. Math. Phys. 39 (1997), 269-275.

[9] R. M. Kashaev and O. Tirkkonen, A proof of the volume conjecture on torus knots, Zap. Nauchn. Sem. S.-Peterburg. Otdel. Mat. Inst. Steklov. (POMI) 269 (2000), 262-268, 370 .

[10] R. Kirby and P. Melvin, The 3-manifold invariants of Witten and ReshetikhinTuraev for $\operatorname{sl}(2, \mathbf{C})$, Invent. Math. 105 (1991), 473-545. 
[11] G. Masbaum, Skein-theoretical derivation of some formulas of Habiro, Algebr. Geom. Topol. 3 (2003), 537-556, arXiv:math.GT/0306345.

[12] H. Murakami, The asymptotic behavior of the colored Jones function of a knot and its volume, in: Proc. of Art of Low Dimensional Topology VI, T. Kohno (ed.), 2000, 87-96, arXiv:math.GT/0004036.

[13] -, Optimistic calculations about the Witten-Reshetikhin-Turaev invariants of closed three-manifolds obtained from the figure-eight knot by integral Dehn surgeries, Sūrikaisekikenkyūsho Kōkyūroku 1172 (2000), 70-79.

[14] - Kashaev's invariant and the volume of a hyperbolic knot after Y. Yokota, in: Physics and Combinatorics 1999 (Nagoya), World Sci., River Edge, NJ, 2001, 244272.

[15] - Some limits of the colored Jones polynomials of the figure-eight knot, Kyungpook Math. J. 44 (2004), 369-383, arXiv:math.GT/0308002.

[16] H. Murakami and J. Murakami, The colored Jones polynomials and the simplicial volume of a knot, Acta Math. 186 (2001), 85-104.

[17] H. Murakami, J. Murakami, M. Okamoto, T. Takata, and Y. Yokota, Kashaev's conjecture and the Chern-Simons invariants of knots and links, Experiment. Math. 11 (2002), 427-435.

[18] H. Murakami and Y. Yokota, The colored Jones polynomials of the figure-eight knot and its Dehn surgery spaces, arXiv:math.GT/0401084.

[19] W. D. Neumann and D. Zagier, Volumes of hyperbolic three-manifolds, Topology 24 (1985), 307-332.

[20] N. Reshetikhin and V. G. Turaev, Invariants of 3-manifolds via link polynomials and quantum groups, Invent. Math. 103 (1991), 547-597.

[21] D. Thurston, Hyperbolic volume and the Jones polynomial, lecture notes, École d'été de Mathématiques "Invariants de nœuds et de variétés de dimension 3", Institut Fourier, 1999.

[22] W. P. Thurston, The Geometry and Topology of Three-Manifolds, electronic version, 2002, http://www.msri.org/publications/books/gt3m/.

[23] -, Three-Dimensional Geometry and Topology. Vol. 1, Princeton Math. Ser. 35, Princeton Univ. Press, Princeton, NJ, 1997.

[24] Y. Yokota, On the volume conjecture for hyperbolic knots, arXiv:math.QA/0009165.

[25] —, On the volume conjecture of hyperbolic knots, in: Knot Theory-dedicated to Professor Kunio Murasugi for his 70th birthday, M. Sakuma (ed.), 2000, 362-367.

[26] - - On the potential functions for the hyperbolic structures of a knot complement, Geom. Topol. Monogr. 4 (2002), 303-311.

[27] - From the Jones polynomial to the A-polynomial of hyperbolic knots, Interdiscip. Inform. Sci. 9 (2003), 11-21.

[28] T. Yoshida, The $\eta$-invariant of hyperbolic 3-manifolds, Invent. Math. 81 (1985), $473-514$.

[29] D. Zagier, The remarkable dilogarithm, J. Math. Phys. Sci. 22 (1988), 131-145.

Department of Mathematics

Tokyo Institute of Technology

Oh-okayama, Meguro, Tokyo 152-8551, Japan

E-mail: starshea@tky3.3web.ne.jp

Received 15 March 2004;

in revised form 20 November 2004 